\title{
THE ESSENTIAL CLOSURE OF $C(X)$
}

\author{
MARLOW ANDERSON
}

\begin{abstract}
Each archimedean l-group admits a unique essential closure, which is the l-group of continuous almost finite real-valued functions on some Stonean space; thus the l-group $C(X)$ of real-valued continuous functions on a topological space $X$ admits such an essential closure. In this note we will construct a natural embedding of $C(X)$ into its essential closure, making explicit the topological relationship between $X$ and the appropriate Stonean space.
\end{abstract}

1. Preliminaries. Throughout this paper $G$ will denote an archimedean lattice-ordered group ( $l$-group). A general reference on $l$-groups is [2]. If $G$ is an $l$-subgroup of an $l$-group $H$, then $G$ is large in $H$ (or $H$ is an essential extension of $G$ ) if $C \cap G \neq 0$, for each convex $l$-subgroup $C$ of $H$. An archimedean $l$-group is essentially closed if it admits no proper archimedean essential extensions; $H$ is an essential closure of $G$ if $H$ is essentially closed and an essential extension of $G$. That each archimedean $l$-group admits a unique essential closure is due to Conrad [5]; this closure is of the form $D(Y)$, where $Y$ is a Stonean space (that is, compact Hausdorff and extremally disconnected). Here $D(Y)=\left\{f: Y \rightarrow \mathbf{R}^{*}: f^{-1}(\mathbf{R})\right.$ is dense in $Y$ and $f$ is continuous $\}$, where $\mathbf{R}^{*}=\mathbf{R} \cup\{ \pm \infty\}$ is the two-point compactification of the real numbers $\mathbf{R}$. Bernau [1] first showed that any archimedean l-group could be embedded into such an l-group.

For $K \subseteq G$, let

$$
K^{\prime}=\{g \in G:|g| \wedge|x|=0, \text { for } x \in K\} .
$$

Then

$$
P(G)=\left\{C^{\prime \prime}: C \text { is a convex } l \text {-subgroup of } G\right\}
$$

is the set of polars of $G ; P(G)$ is a subset of the set of convex l-subgroups of $G$, and is a complete Boolean algebra, with set-theoretic intersection for meet, and ' for complementation. If $H$ is archimedean and $G \subseteq H$, then $G$ is large in $H$ precisely when the natural intersection map $C \rightarrow C \cap G$ is a Boolean algebra isomorphism between $P(H)$ and $P(G)$ [4].

Given a Tychonoff space $X$, let $\Theta X$ be the set of all regularly open ultrafilters on $X$. If $U$ is a regularly open subset of $X$, let $\Theta(U)=\{p \in \Theta X$ : $U \in p\}$. The set of all such $\Theta(U)$ forms a base for the Stone topology on $\Theta X$, which makes $\Theta X$ a Stonean space [6]. Furthermore, $\vartheta: R(X) \rightarrow \mathscr{R}(\Theta X)$

Received by the editors March 14, 1979.

AMS (MOS) subject classifications (1970). Primary 06A60; Secondary 54C40. 
is a Boolean algebra isomorphism between the regularly open subsets of $X$ and of $\Theta X[3$, p. 40]. Now let $\omega X=\{p \in \Theta X: p$ is fixed $\}$, which is called the absolute of $X$. Then $\omega X$ is a dense subset of $\Theta X[6]$, and so $\beta(\omega X)=\Theta X$. Define $\pi: \omega X \rightarrow X$ by letting $\pi(p)$ be the (unique) point to which $p$ converges. Then $\pi$ is a continuous function [6].

2. The embedding. We will need the following result which characterizes polars topologically:

Proposition. Let $X$ be a Tychonoff space. Let $G=C(X)($ or $D(X)$, where $X$ is Stonean). Then the map $\tau(X): P(G) \rightarrow \Re(X)$ defined by

$$
\tau(X)(C)=\text { Interior(Closure }\{x \in X: f(x) \neq 0 \text {, some } f \in C\} \text { ) }
$$

is a Boolean algebra isomorphism.

This proposition is well known and its proof will be omitted.

THEOREM. If $X$ is a Tychonoff space, then $\alpha: C(X) \rightarrow D(\Theta X)$, defined so that the following diagram commutes for all $f \in C(X)$, is a large l-embedding of $C(X)$ into its essential closure:

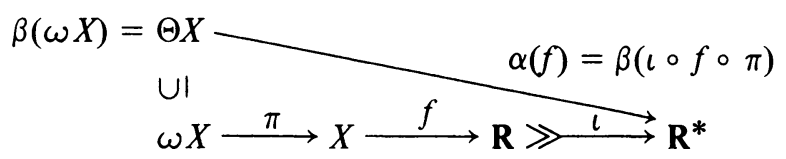

Proof. Since $\alpha(f)^{-1}(\mathbf{R})$ is an open subset of $\Theta X$ which contains $\omega X$ and is consequently dense, $\alpha(f)$ is an element of $D(\Theta X)$. Given $f, g \in C(X), \alpha(f)+$ $\alpha(g)$ is defined as the unique extension of $\alpha(f)|U+\alpha(g)| U$ to $\Theta X$, where $U=\alpha(f)^{-1}(\mathbf{R}) \cap \alpha(f)^{-1}(\mathbf{R})$, an open dense set. (This unique extension exists because $X$ is Stonean.) But $\alpha(f)+\alpha(g)$ then agrees with $\alpha(f+g)$ on $\omega X$, a dense subset of $\Theta X$, and so $\alpha(f)+\alpha(g)=\alpha(f+g)$. A similar argument shows that $\alpha$ preserves the lattice operations. Since $\alpha$ is clearly monic, it remains to show that $C(X)$ (identified now with $\alpha(C(X))$ )) is large in $D(\Theta X)$. But

$$
\tau(X)^{-1} \Theta^{-1} \tau(\Theta X): P(D(\Theta X)) \rightarrow P(C(X))
$$

is a Boolean algebra isomorphism, and

$$
\tau(X)^{-1} \mathcal{O}^{-1} \tau(\Theta X)(C)=C \cap C(X) .
$$

\section{REFERENCES}

1. S. Bernau, Unique representations of lattice groups and normal archimedean lattice rings, Proc. London Math. Soc. 15 (1965), 599-631.

2. A. Bigard, K. Keimel and S. Wolfenstein, Groupes et anneaux réticulés, Springer-Verlag, Berlin, 1977.

3. W. Comfort and S. Negrepontis, The theory of ultrafilters, Springer-Verlag, New York, 1974. 
4. P. Conrad, The lateral completion of a lattice-ordered group, Proc. London Math. Soc. 19 (1969), 444-486.

5. The essential closure of an archimedean lattice-ordered group, Duke Math. J. 38 (1971), 151-160.

6. S. Iliadis and S. Fomin, The method of centred systems in the theory of topological spaces, Uspehi Mat. Nauk 21 (1966), 47-76 = Russian Math. Surveys 21 (1966), 37-62.

Department of Mathematics, Universtty of Kansas, LaWrence, Kansas 66045

Current address: Mathematics Department, Indiana University-Purdue University at Fort Wayne, 2101 Coliseum Boulevard East, Fort Wayne, Indiana 46805 